Abstracted/indexed in Academic Search Complete, Asia Journals Online, Bangladesh Journals Online, Biological Abstracts, BIOSIS Previews, CAB
Abstracts, Current Abstracts, Directory of Open Access Journals, EMBASE/Excerpta Medica, Google Scholar, HINARI (WHO), International

Pharmaceutical Abstracts, Open J-gate, Science Citation Index Expanded, SCOPUS and Social Sciences Citation Index;

\title{
Cyclooxygenase-2 expression and its association with vascular endothelial growth factor, microvessel density and clinicopatho- logic characteristics of colorectal carcinoma
}

\author{
Zhao-Yang Qin'1, Xiao-Yan Guo'1, Jing-Zhong $\mathrm{Xu}^{2}$ and Gang-Ping Wang3 \\ ${ }^{1}$ Department of General Surgery, Rizhao People's Hospital, Rizhao 276 826, China; ${ }^{2}$ Department of Traditional \\ Chinese Medicine, Jufeng Hospital, Rizhao 276 826, China; ${ }^{3}$ Department of Pathology, Rizhao People's Hospital, \\ Rizhao 276 826, China.
}

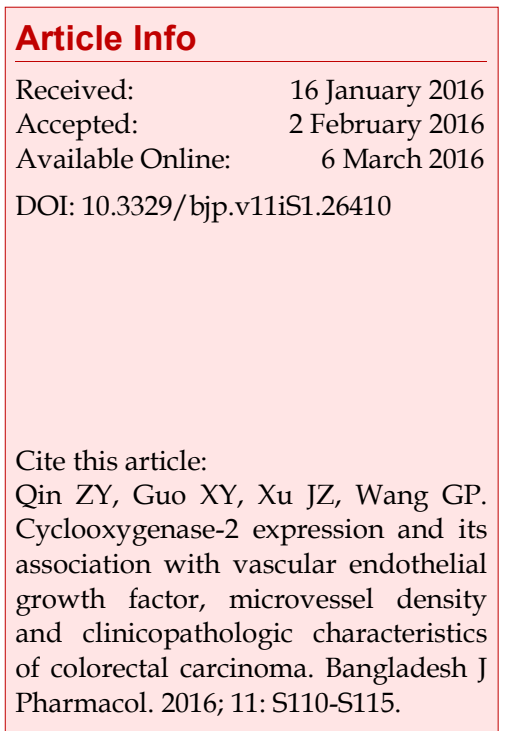

\begin{abstract}
The aim of this study was to investigate the expression of cyclooxygenase type-2 (COX-2) and its association with angiogenesis and clinicopathologic characteristics of colorectal carcinoma (CRC). COX-2 expression was detected by means of immunohistochemistry in a series of human tissue samples with CRC ( $n=120)$, dysplasia tissue closely adjacent to carcinomas $(n=40)$ and normal colorectal mucosa $(n=40)$, and their expressions association with vascular endothelial growth factor (VEGF), CD31-labeled micorvessel density (MVD) in CRC and other clinicopathologic characteristics were investigated. The expression of COX-2 in CRC tissues (78.3\%) was obviously higher than that in adjacent tissue and normal mucosal tissue $(p<0.01)$. COX-2 expression was correlated significantly with the grading, advanced cancer, Dukes stage and lymph node metastasis, distant metastasis and VEGF $(p<0.05)$. Our result demonstrates that COX-2 expression was significantly higher in earlier stages of CRC. It can be suggested that COX-2 expression may be important in the initial development of CRC. The findings of the present study suggest that COX-2 overexpression in CRC may be considered as a negative prognostic marker.
\end{abstract}

\section{Introduction}

Colorectal carcinoma (CRC) is a malignant epithelial tumor that penetrates through muscularis mucosae into submucosa. It varies considerably throughout the world, being one of the leading cancer sites in the developed countries (Al-Maghrabi et al., 2012; Dixon et al., 2013; Peng et al., 2013). Carcinoma prognosis is directly related to factors such as tumor size, lymph node involvement, presence of distant metastasis, and so on (Lavalle et al., 2009). Large tumors with lymph

This paper was presented in the 3rd International Conference on Biomedicine and Pharmaceutics in Zhuhai, China, on December 11-13, 2015. node involvement or distant metastases indicate a poor prognosis, worse overall and disease-free survival.

Cellular conditions such as hypoxia lead to increased cyclooxygenase-2 (COX-2) expression. COX-2 is an indu -cible enzyme that interferes with tumor development and angiogenesis, related to the inhibition of apoptosis through inhibition of the proapoptotic bax protein and overexpression of the antiapoptotic bcl-2 protein (Miglietta et al., 2010; Yi et al., 2014).

Recently, it has been reported that COX-2 is implicated in carcinogenesis and tumor progression in many cancers, including CRC. The aberrantly express COX-2, and that raised tissue levels of COX-2 may have 
prognostic value. Patients expressing high levels of COX-2 can develop local recurrence, and have reduced disease-free and disease-related overall survival (Dixon et al., 2013; Miladi-Abdennadher et al., 2012). However, other studies did not report significant correlations between COX-2 expression and histopathological parameters or disease-free survival in breast cancer (Kelly et al., 2003; Ranger et al., 2004).

Angiogenesis is essential for the growth and metastasis of major solid tumors (Kopczyńska and Makarewicz, 2012). High levels of angiogenic factors and histologic evidence of increased tumor neovascularization through microvessel density (MVD) measurement are considered to have important prognostic value for various solid tumors, with increased risk of disease dissemination and tumor mass development. MVD may be an indicator of malignancy and represent the angiogenic potential of the neoplasm (Kopczyńska and Makarewicz, 2012). However, the precise implication of COX-2 and angiogenesis in CRC and their associations with invasion and metastasis of CRC has not yet been studied.

In the present study, we investigated the significance of COX-2 and vessels recognized by CD31 as endothelial markers by immunohistochemical staining in 120 cases of curatively resected CRC and analyzed the relationship between COX-2, ki-67, MVD expression with standard prognostic parameters, such as size, grade, metastases, and patient survival, in order to study the clinical and pathological characteristics associated with invasive CRC.

\section{Materials and Methods}

\section{Patients}

In this study, the expression of COX-2, VEGF, MVD and ki-67 were measured by immunohistochemical ultrasensitive method (S-P method) staining in 120 cases of primary CRC, 40 cases of dysplasia tissue closely adjacent to carcinomas, and 40 cases of normal colorectal mucosa specimens with complete clinical data from 2002 to 2015. All the clinical and follow-up information were reviewed and evaluated and their relationship with well-known clinicopathological factors such as tumor size, grade, lymph node status and stage were investigated in CRC patients. One hundred twenty patients diagnosed CRC were collected during excision surgery at Rizhao People's Hospital.

The patients diagnosed CRC had not been treated with hormone endocrine therapy, anti-neoplastic chemotherapy or radiotherapy during the last six months. The COX-2 expression assessment was done by immunohistochemical methods in $\mathrm{CRC}$, and their relationship with clinical-pathological parameters including the express of VEGF, ki-67 proliferation index, CD31, and the biomarkers levels of serum biomarkers such as carcinoembryonic antigen (CEA) and malignant tumor specific growth factor (TSGF) and histological grade, region lymph node metastasis, distant metastasis and recurrence on files were also assessed in order to study the clinical and pathological characteristics associated with CRC.

\section{Biomarkers measure}

For TSGF and CEA analysis, $3 \mathrm{~mL}$ heparinized blood was drawn from each individual. The biomarkers levels were detected in 120 cases CRC with electrochemiluminescence method in the clinical laboratory in Rizhao People's Hospital. The cut off values of TSGF and CEA in serum are $70.00 \mathrm{U} / \mathrm{mL}$ and $3.40 \mathrm{ng} / \mathrm{mL}$. For the biomarkers levels, patients are grouped into two groups as normal level or high level peripheral blood, respectively (Wang et al., 2014).

\section{Pathologic study}

All specimens were fixed in formalin and embedded in paraffin. Serial sections $(4 \mu \mathrm{m})$ were depar-affinized in xylene and hydrated through a graded series of ethanol. The specimens were washed in phosphate-buffered saline (PBS) within five minutes and examined under a binocular dissecting microscope. The pathological diagnosis was verified by histological methods independently by two pathologists, and pathological categorization was determined according to the current world health organization classification system diagnostic criteria. According to histological grade they are classified into two groups as Grade (I + II) and Grade III. COX-2, VEGF, ki-67 and CD31 expression assessment was done by immuno-histochemical methods. Immunoreactions were process-ed using the ultrasensitive S-P kit (Maixin-Bio Co., China) according to the manufacturer's instructions, and signals were visualized using the DAB substrate, which stains the target protein yellow. The negative controls were used. The primary antibody was replaced with PBS, containing $0.1 \%$ bovine serum albumin at the same concentration as the primary antibody. The positive controls were tissues known to express the antigen being studied. COX-2 and VEGF immunoreactivity expression the percentage of cancer cells showed cytoplasmic staining reactivity.

The immunoreactive score (IRS) was obtained by multiplying the percentage of positive cells and the staining intensity. In brief, a proportion score was assigned that represents the estimated proportion of positive tumor cells on the entire slide. For each histological section, the percentage of positive cells was scored as $0(<5 \%), 1(6 \%-25 \%), 2(26 \%-50 \%), 3(51 \%-$ $75 \%)$ and $4(>75 \%)$, and the staining intensity was scored as 0 (negative), 1 (weak), 2 (moderate) and 3 (strong). Immunohistochemical results with an IRS of 0 were considered negative, 1-4 weak positive, 5-8 
moderate positive and 9-12 strong positive. For ki-67 expression the percentage of cancer cells showing a nuclear reactivity was recorded after inspection of all optical fields at 200 and the mean value was used to score each case. The MVD immunohistochemistry with CD31 was evaluated under light microscopy according to the procedure described elsewhere (Kopczyńska and Makarewicz, 2012). CD31 positivity was indicated by membrane staining. To analyze MVD-CD31, structures with or without lumens that were positive for CD31 were considered as individual vessels. Briefly, after scanning the sections at low magnifications, five tumor areas with the greatest number of distinctly highlighted micovessels were selected as hot spots. The number of vessels was counted in the highlighted micovessels at high magnifications (400×), from which the median was obtained. Each brown-stained endothelial cell or endothelial cell cluster, which was clearly separate from the adjacent micovessels, tumor cells and connective tissue elements were considered a single, countable microvessel. Areas of fibrosis, necrosis and inflammation, and vessels with muscular walls were not counted. Assessment of the staining was evaluated by two independent pathologists without knowledge of the clinical status of the patients.

\section{Statistical analysis}

SPSS ver 17.0 statistical software (SPSS inc., USA) was used to detect the data. Measurement data expressed with mean and standard deviation (mean \pm SD) between groups was compared with $t$-test, while enumeration data with $c^{2}$ test. The $p$ value was considered to be significant if less than 0.05 .

\section{Results}

\section{Clinical features and follow-up date}

In 120 cases of CRC patient, the age was 22-79 years (median 53 years); 81 cases were male, 39 cases were female. Tumor size was $0.2 \sim 9.5 \mathrm{~cm}$. 20 cases were dukes A, 36 cases were dukes B, 50 cases dukes C, and 14 cases dukes D. 42 cases were lost to follow-up, 78 cases were followed-up, including 18 patients alive, 54 patients died of CRC, 6 patients died of other reasons.

COX-2 express and relationship with clinicopathological factors

\section{COX-2 express in different groups}

In CRC tissues, cancer adjacent tissues and mucosal tissues, COX-2 expression was located in the tumor cell cytoplasm. The expressions of COX-2 in different groups were shown in Table I. Immunohistochemical analysis revealed COX-2 expression in CRC tissue was significantly higher than that in dysplasia and normal colorectal mucosa specimens $(\mathrm{p}<0.05)$. The expression of COX-2 in the examined CRC tumors tissue was $78.3 \%$ (94/120) with moderate positive to strong some degree of positivity. In cancer adjacent tissues and mucosal tissues, COX-2 was weekly expressed only $25.0 \%(10 / 40)$ and $10.0 \%(4 / 40)$, respectively. The expression of COX-2 in colorectal cancer tissues obviously was higher than the expression in adjacent tissue organizations $\left(\chi^{2}=18.755, \mathrm{p}=0.000\right)$ and normal mucosal tissue $\left(\chi^{2}=29.511, \mathrm{p}=0.000\right)$, the difference was statistically significant $(\mathrm{p}<0.01$, respectively), but COX-2 in cancer in expression than in heterogeneous organization of bowel mucosa tissue of expression, but the difference of COX-2 expression was not statistically significant $\left(\chi^{2}=0.693, \mathrm{p}=0.405\right)$.

\section{COX-2 expression in CRC different histological grade} groups

The expression of COX-2 in the high grade carcinoma $(67.6 \%)$ was higher than that in low grade cancer $(95.7 \%)$, the difference was statistically significant $\left(\chi^{2}=\right.$ 6.591, $\mathrm{p}=0.010$; Table II).

\section{COX-2 expression in CRC different Dukes staging groups}

Comparison with CRC dukes A and B stage group, the difference of COX-2 expression in CRC with dukes C and $\mathrm{D}$ group was statistically significant $\left(\chi^{2}=6.104, \mathrm{p}=\right.$ $0.014)$. The difference wasn't statistically significant between CRC dukes A and B groups $\left(\chi^{2}=0.124, p=\right.$ 0.727), between CRC dukes $C$ and D groups $\left(\chi^{2}=0.013\right.$, $\mathrm{p}=0.910)$, as shown in Table II.

Table I

COX-2 expression in different groups

\begin{tabular}{|c|c|c|c|c|c|}
\hline \multirow[t]{2}{*}{ Different tissues } & \multirow[t]{2}{*}{$\mathrm{n}$} & \multicolumn{4}{|c|}{ COX-2 } \\
\hline & & Positive & Negative & $x^{2}$ & $\mathrm{p}$ value \\
\hline Cancer tissues & 120 & 94 & 26 & - & - \\
\hline Adjacent tissue & 40 & 10 & 30 & $18.755^{a}$ & $0.000^{\mathrm{a}}$ \\
\hline Normal tissue & 40 & 4 & 36 & $29.511^{b}$ & $0.000^{\mathrm{b}}$ \\
\hline
\end{tabular}

aComparison between cancer and adjacent tissues; bcomparison between cancer and normal mucosal tissues, the difference was statistically significant $(\mathrm{p}<0.01$, respectively); Comparison between cancer adjacent tissues and normal mucosal tissues, the difference wasn't statistically significant $\left(\chi^{2}=0.693, p=0.405\right)$ 


\begin{tabular}{|c|c|c|c|c|c|}
\hline \multicolumn{6}{|c|}{ Table II } \\
\hline \multicolumn{6}{|c|}{ COX-2 expression in CRC different groups } \\
\hline \multirow[t]{2}{*}{ Biological parameters } & \multirow[t]{2}{*}{$\mathrm{n}$} & \multicolumn{2}{|c|}{ COX-2 } & \multirow[t]{2}{*}{$x^{2}$} & \multirow[t]{2}{*}{$\mathrm{p}$ value } \\
\hline & & Positive & Negative & & \\
\hline \multicolumn{6}{|l|}{ Histological grade } \\
\hline Low & 74 & 50 & 12 & 6.591 & 0.010 \\
\hline High & 46 & 44 & 1 & & \\
\hline \multicolumn{6}{|l|}{ Dukes stage } \\
\hline A \& B & 56 & 36 & 20 & 0.124 & 0.014 \\
\hline$C \& D$ & 64 & 58 & 6 & 6.104 & \\
\hline \multicolumn{6}{|l|}{ Infiltration } \\
\hline Early cancer & 18 & 8 & 10 & 4.925 & 0.027 \\
\hline Advanced cancer & 102 & 86 & 16 & & \\
\hline \multicolumn{6}{|l|}{ Lymph node status } \\
\hline Absent & 56 & 36 & 20 & 6.104 & 0.014 \\
\hline Present & 64 & 58 & 6 & & \\
\hline
\end{tabular}

\section{COX-2 expression in CRC different infiltrating depth}

The expression of COX-2 in advanced cancer was significantly raised $(44.4 \%$ vs $84.3 \%)$, and with a statistical significance than that in early cancer $\left(x^{2}=\right.$ 4.925, $\mathrm{p}=0.027), \mathrm{p}<0.05$.

\section{COX-2 express in different lymph node status groups}

The expression of COX-2 in the group with lymph node metastasis $(90.6 \%)$ was higher than that in the group without lymph node metastasis $(64.3 \%)\left(\chi^{2}=6.104, \mathrm{p}=\right.$ $0.014)$, and there were significantly statistically significant between the two groups, $\mathrm{p}<0.05$.

\section{Comparison of biomarker levels to COX-2 express}

The TSGF and CEA serum levels in COX-2 positive and negative groups in 120 cases of CRC patients were shown in Table III. There were no significantly difference among the two groups ( $p>0.05$, respectively ).

\section{COX-2 and VEGF expression and CD31-labeled MVD assessment in CRC}

The positive rate of VEGF expression and MVD in CRC $(65.0 \%, 50.9 \pm 21.4)$ was higher than that in colorectal dysplasia tissue $(15.0 \%, 21.3 \pm 11.6)$, and normal colorectal mucosa $(15.0 \%, 20.8 \pm 10.2)(p<0.05)$. The VEGF expression in COX-2 positive group was higher

Table III

\begin{tabular}{|lccc|}
\hline \multicolumn{4}{|c|}{ Table III } \\
\hline \multicolumn{4}{|c|}{ Comparison of biomarker levels to } \\
COX-2 expression \\
\hline COX-2 express & $\mathrm{n}$ & TSGF $(\mathrm{U} / \mathrm{mL})$ & CEA $(\mathrm{U} / \mathrm{mL})$ \\
\hline Positive & 94 & $168.5 \pm 34.7$ & $79.9 \pm 32.5$ \\
Negative & 26 & $157.4 \pm 33.7$ & $74.2 \pm 30.0$ \\
\hline
\end{tabular}

than those in COX-2 negative group, there were slightly correlated with CD31 staining MVD, MVD in COX-2 positive group was higher than those in COX-2 negative group, but there were no significantly statistically significant between the two groups $(\mathrm{p}>0.05)$.

\section{Survival analysis}

Patients were monitored for survival from 2006 to 2015 through telephone communication and periodic returns to the rizhao people's hospital. Follow-up information was available for 78 of the patients. 17 patients relapsed, and 54 patients died during the course of the follow-up period. Overall survival was defined as the period (months) between surgical removal and death caused by the neoplastic process. The patients whose deaths occurred from any other cause were not taken into account. Patients were censored if the follow-up period was less than 6 months. For COX-2 expression, the patients were divided into negative and positive two groups. For MVD, the patients were also classified two groups according to the median values: Median 30 or below, median more than 30 . Considering only the COX-2 expression, survival was found to be longer in negative group than that in positive group, and there were slightly statistically significant between the two groups $(\mathrm{p}<0.05)$. Other factors which may be associated with COX-2 in CRC, which should be investigated in further studies.

\section{Discussion}

In our study, there was positive correlation in overexpression of COX-2 in CRC with histological high grade (III), lymph node metastasis and CRC dukes A and $\mathrm{B}$ stage group. There was positive correlation in overexpression of COX-2 in CRC with histological high 
grade (III), lymph node metastasis and CRC dukes A and $\mathrm{B}$ stage group. COX-2 catalyzes the conversion of arachidonic acid to PGE2 and enhances the metastatic phenotype tumors. PGE2, the catalytic product of COX2 , may promote tumor development and angiogenesis. It has been investigated in several human cancers and also correlated with the evolution of the disease. In our study, our results revealed that patients with increased COX-2 expression have shorter survival times. A slightly correlation between COX-2 expression and prognostic factors in CRC suggesting that increased COX-2 expression was related to worse prognosis, as observed in the survival analysis in our study. We suggest that the difference between COX-2 expression in the CRC may related to the differentiated behavior of these tumors, confirming the association between the COX-2 expression and disease aggressiveness. Besides showing shorter overall survival, it was verified that COX-2 expression correlated sight positively with CRC, suggesting a worse prognosis. In our study, we have observed shorter survival in patients whose tumors expressed more COX-2. Therefore, there are possibility of using COX-2 inhibitor anti-inflammatory drugs to treat mammary tumors.

Angiogenesis is essential for the growth and metastasis of major solid tumors and has been correlated with prognosis in human cancer (Kopczyńska and Makarewicz, 2012; Thielemann et al., 2013; Wang et al., 2014). VEGF capable of promoting angiogenesis exerts an important effect in the process of genesis, development, metastasis and recurrence of various tumors. In the process of tumor genesis and development, tumor regenerative capillaries capable of providing nutrients for tumor cells and favorable conditions for distal metastasis are the precondition to induce the local growth, infiltration and distal metastasis of malignant tumors, hence, how to inhibit tumor angiogenesis is a new research hotspot at present (Thorat et al., 2013). VEGF, one of the key factors to promote tumor angiogenesis and with the strongest function and highest specificity, can not only promote the proliferation of endothelial cells, but also regulate and participate in angiogenesis (Thorat et al., 2013). Due to an intimate association with genesis, development, metastasis and infiltration, it is an important indicator to judge the cancer metastasis and infiltration in clinic (Koyama et al., 2010; Wang et al., 2013). In the study, VEGF expression in CRC (65.0\%) was higher than that in colorectal dysplasia tissue $(15.0 \%)$ and normal colorectal mucosa $(15.0 \%)(\mathrm{p}<0.05)$.

We also investigated the prognostic significance of CD31 and MVD assessed based on the number of CD31 -positive vessel in various tissues of CRC and epithelium adjacent to the lesion. In our study, we investigated the prognostic significance of MVD assessed based on the number of CD31-positive vessel in various tissues of CRC and benign lesions and epithelium adjacent to the lesion. The results showed that MVD in CRC was higher than that in colorectal dysplasia tissue and normal colorectal mucosa $(\mathrm{p}<$ 0.05). The MVD in COX-2 positive group was higher than that in COX-2 negative group, there were slightly correlated with MVD. Tumor angiogenesis and its clinical significance have been studied in a variety of neoplasms. Angiogenesis contributes to the pathogenesis of various cancer, and microvessel density may improve our ability to predict CRC extension. Microvessel densities are significantly greater in the primary tumors of patients with metastatic disease than in those without metastases. When we assessed overall survival and angiogenesis, our results suggesting that angiogenesis is essential for the development and evolution of neoplastic disease, resulting in shorter overall survival and worse prognosis. Our results support that MVD recognized by CD31 is closely relevant to lymph node metastasis and recurrence of CRC, and act as a valuable indicators of prognosis. MVD-CD31 may be useful as a predictor for the recurrence of CRC and may have a specific association with the development of locoregional. In CRC, the potential application of MVD recognized by CD31 as tumor angiogenesis markers, useful for cancer diagnostics and clinical application, is anticipated. We suggest that the increased expression of MVD in tumors indicates a higher risk of development and dissemination of the disease.

\section{Conclusion}

COX-2 expression was significantly higher in earlier stages of CRC. It can be suggested that COX-2 expression may be important in the initial development of CRC. The findings of the present study suggest that COX-2 overexpression in CRC may be considered as a negative prognostic marker.

\section{Ethical issue}

The study protocol was endorsed by the Medical Ethical Committee of the Hospital; besides, each parti-cipant provided informed consent.

\section{Conflict of Interest}

The author confirms that this article content has no conflict of interest.

\section{References}

Al-Maghrabi J, Buhmeida A, Emam E, Syrjänen K, Sibiany A, Al-Qahtani M, Al-Ahwal M. Cyclooxygenase-2 expression as a predictor of outcome in colorectal carcinoma. World J Gastroenterol. 2012; 18: 1793-99. 
Dixon DA, Blanco FF, Bruno A, Patrignani P. Mechanistic aspects of COX-2 expression in colorectal neoplasia. Recent Results Cancer Res. 2013; 191: 7-37.

Kelly LM, Hill AD, Kennedy S, Connolly EM, Ramanath R, Teh S, Dijkstra B, Purcell R, McDermott EW, O'Higgins N. Lack of prognostic effect of Cox-2 expression in primary breast cancer on short-term follow-up. Eur J Surg Oncol. 2003; 29: 707-10.

Kopczyńska E, Makarewicz R. Endoglin: A marker of vascular endothelial cell proliferation in cancer. Wspolczesna onkol. 2012; 16: 68-71.

Koyama Y, Okayama H, Kumamoto K, Saito K, Nakamura I, Ohki S, Takenoshita S. Overexpression of endoglin (CD105) is associated with recurrence in radically resected gastric cancer. Exp Ther Med. 2010; 1: 627-33.

Lavalle GE, bertagnolli AC, tavares WLF, Cassali GD. Cox-2 expression in canine mammary carcinomas: Correlation with angiogenesis and overall survival. Vet Pathol. 2009; 46: 127580.

Miglietta A, Toselli M, Ravarino N, Vencia W, Chiecchio A, Bozzo F, Motta M, Torchio B, Bocca C. COX-2 expression in human breast carcinomas: Correlation with clinicopathological features and prognostic molecular markers. Expert Opin Ther Targets. 2010; 14: 655-64.

Miladi-Abdennadher I, Abdelmaksoud-Dammak R, AyedGuerfali DB, Ayadi L, Khabir A, Amouri A, Frikha F, Tahri N, Ellouz S, Frikha M, Sellami-Boudawara T, MokdadGargouri R. Expression of COX-2 and E-cadherin in Tunisian patients with colorectal adenocarcinoma. Acta Histochem. 2012; 114: 577-81.

Peng L, Zhou Y, Wang Y, Mou H, Zhao Q. Prognostic significance of COX-2 immunohistochemical expression in colorectal cancer: A meta-analysis of the literature. PLoS One.
2013; 8: e58891.

Ranger GS, Jewell A, Thomas V, Mokbel K. Elevated expression of cyclooxygenase-2 in breast cancer and ductal carcinoma in situ has no correlation with established prognostic markers. J Surg Oncol. 2004; 88: 100-03.

Thielemann A, Baszczuk A, Kopczyński Z, Kopczyński P, Grodecka-Gazdecka S. Clinical usefulness of assessing VEGF and soluble receptors sVEGFR-1 and sVEGFR-2 in women with breast cancer. Ann Agric Environ Med. 2013, 20: 293-97.

Thorat D, Sahu A, Behera R, Lohite K, Deshmukh S, Mane A, Karnik S, Doke S, Kundu GC. Association of osteopontin and cyclooxygenase-2 expression with breast cancer subtypes and their use as potential biomarkers. Oncol Lett. 2013; 6: 1559-64.

Wang G, Qin Y, Zhang J, Zhao J, Liang Y, Zhang Z, Qin M, Sun Y. Nipple discharge of CA15-3, CA125, CEA and TSGF as a new biomarker panel for breast cancer. Int J Mol Sci. 2014; 15: 9546-65.

Wang GP, Qin MH, Liang YA, Zhang H, Zhang ZF. The significance of biomarkers in nipple discharge and serum in diagnosis of breast cancer. In: Advanced Engineering and Technology. Liquan Xie, Dianjian Huang (eds.). CRC Press, 2014, pp 665-71.

Wang JJ, Sun XC, Hu L, Liu ZF, Yu HP, Li H, Wang SY, Wang DH. Endoglin (CD105) expression on microvessel endothelial cells in juvenile nasopharyngeal angiofibroma: Tissue microarray analysis and association with prognostic significance. Head Neck. 2013; 35: 1719-25.

Yi C, Zhang Y, Yu Z, Xiao Y, Wang J, Wang J, Qiu H, Yu W, Tang R, Yuan Y, Guo W, Deng W. Melatonin enhances the anti-tumor effect of fisetin by inhibiting COX-2/iNOS and NF-kB/p300 signaling pathways. PLoS ONE. 2014; 9: e99943.

\footnotetext{
Author Info

Gang-Ping Wang (Principal contact)

e-mail: wgprzph93@126.com; Tel: +86-633-3365056
} 


\section{Your feedback about this paper}

1. Number of times you have read this paper 0

2. Quality of paper
Excellent
$\bigcirc$ Good
Moderate
Not good

3. Your comments

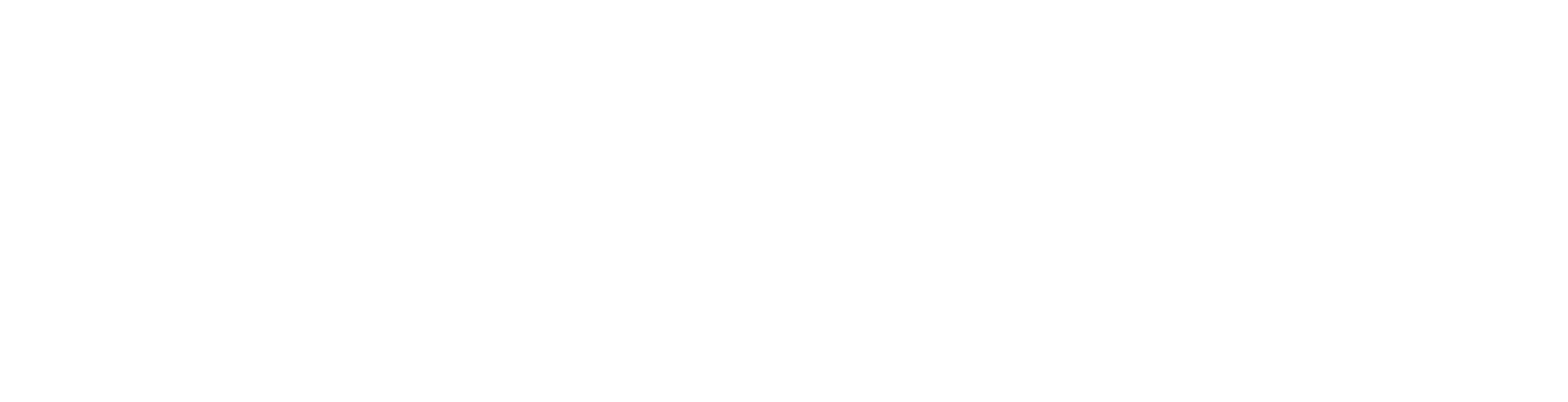

